\title{
Reseña
}

\section{Comunicación y salud, un campo emergente en Latinoamérica}

https://doi.org/10.18566/comunica.n45.a07

Recibido: 29 de abril de 2021

Aceptado: 31 de mayo de 2021

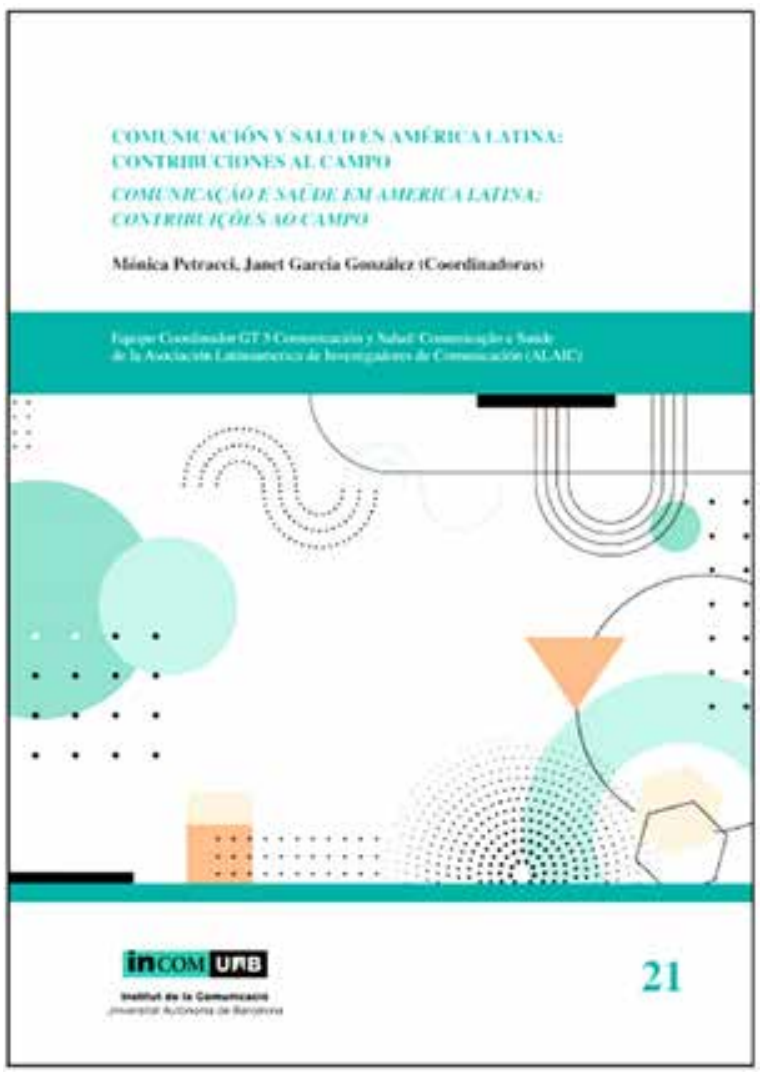

Disponible para su descarga en https://ddd.uab.cat/pub/ llibres/2020/233410/ComunicacionySalud-ebook_21.pdf
Recientemente ha aparecido el libro Comunicación y salud en América Latina: contribuciones al campo, editado por Mónica Petracci y Janet García (2020). Fue publicado, en acceso libre, por InComUAB Publicacions, ${ }^{1}$ con objetivos, según sus editoras, académicos e institucionales: por un lado, "apuntan al fortalecimiento y visibilidad del campo Comunicación y Salud, del Grupo de Trabajo (GT) en Comunicación y Salud de la Asociación Latinoamericana de Investigadores en Comunicación (ALAIC) y de las relaciones entre los y las investigadores de la región"; y, por otro, esta obra "permitirá que la producción académica discutida en los Congresos de ALAIC sea, en primer lugar, difundida entre las y los investigadores del campo a la vez que contribuya a fortalecer la identidad del GT de una institución dedicada a reflexionar sobre la comunicación en la región". comunicación

número 45

Julio - diciembre

2021 | pp. $79-85$

\section{José Luis Terrón}

Docente e investigador de la UAB y miembro del Instituto de la Comunicación de la Universidad Autónoma de Barcelona (InCom-UAB). Doctor en Ciencias de la Información.

joseluis.terron@uab.cat
1 https://ddd.uab.cat/ pub/llibres/2020/233410/ ComunicacionySalud-ebook_21. pdf 
Dos objetivos con los que se pretende lograr estas finalidades: compilar, vindicar, afianzar y diseminar los estudios sobre comunicación y salud realizados en Latinoamérica. Y adelantamos al lector que esta obra no solo persigue esas finalidades, sino que logra ser una herramienta para su consecución. Por primera vez se publica una obra tan ambiciosa sobre el campo de la comunicación y la salud en la región, por lo que debemos felicitarnos. Aún son escasos los libros sobre este campo en español y portugués, y cada uno que aparece es un pequeño empuje a la consolidación de tales temas.

Pero antes de profundizar, es oportuno reparar en una serie de cuestiones que delimitan la comunicación y la salud, sobre todo para aquellos lectores que por primera vez se acerquen a este campo.

Al igual que Ramírez Leyva (2015) y Navarro (1998), sostenemos que la manera de entender la salud ha ido evolucionando históricamente y, por lo tanto, hay una continua redefinición. Aun en un mismo periodo histórico, no todos entendemos por igual qué es, así que debemos huir de la opción de considerarla como un concepto consensuado, monosémico y aséptico. Esta claridad es fundamental, ya que nuestras reflexiones e investigaciones básicas y aplicadas se verán determinadas por este punto de partida. 0 como escribieron Castiel y Álvarez Dardet (2007), "los discursos acerca de la salud significan modos de pensar, escribir, hablar acerca de la salud y sus prácticas" (2007, p. 462).

En 1946, la Organización Mundial de la Salud (OMS) la definió como "el completo estado de bienestar físico, mental y social y no sólo la ausencia de enfermedad" (Terrón, 2010, p. 79). Esta concepción fue en su momento revolucionaria: no se trataba de una simple oposición a la idea de enfermedad y, a la vez, huía de una definición marcadamente biologicista, marco preeminente hasta entonces. Ahora bien, con el paso de los años se vio que esta definición también promueve una serie de ideas y de prácticas rechazables en sí mismas o por llevar a la confusión. Mencionemos algunas: la idea de salud subyacente aquí es utópica (Huber et al., 2011), promueve la medicalización a la par que el fenómeno del healthism (la obsesión por la salud) y la profusión de los discursos paracientíficos (muchos de ellos mercantiles) para los cuales la salud pasa de ser un fin a ser un argumento para otros fines. Y es que no debemos olvidar la distinción entre culto al cuerpo, cultivo del cuerpo y cuidado del cuerpo (Díaz Rojo et al., 2006, pp. 25-26). Por otro lado, una cosa es la salud y otra la percepción que tenemos sobre ella.

Por nuestra parte, estamos más cómodos con la definición que hace el Hastings Center (2004, p.35): 
La salud se caracteriza por la ausencia de males de consideración y, por tanto, por la capacidad de una persona para perseguir sus metas vitales y desenvolverse adecuadamente en contextos sociales y laborales habituales. Con esta definición queremos subrayar el énfasis tradicional en la integridad y el buen funcionamiento del cuerpo, la ausencia de disfunciones y la consiguiente capacidad de actuar en el mundo.

Ahora bien, siempre y cuando tengamos en cuenta que debemos incluir la salud mental en esta definición.

Sobre qué podemos entender por comunicación y salud, recordemos que Schiavo (2007) la considera una rama del conocimiento multidisciplinaria que abarca desde la promoción de la salud hasta la alfabetización o la educación para la salud. Con pocas palabras, y como la mayoría de las definiciones sobre este campo, su definición viene dada por sus finalidades. Hagamos en este punto un poco de historia.

Desde hace años defendemos que este campo se sostiene sobre dos tradiciones: por un lado, la estadounidense y, por otro, la latinoamericana. En Estados Unidos surge a partir de las necesidades de comunicación entre los profesionales de la salud y los pacientes. De hecho, hoy en día en las revistas internacionales de gran impacto hay más autores que hablan sobre comunicación y salud entre los profesionales de la segunda que entre los comunicólogos. Por otra parte, Luis Ramírez Beltrán (1995) nos recordó que en Latinoamérica debemos buscar su génesis en la comunicación para el desarrollo, sabiendo que esos programas tenían tres patas: la agricultura, la educación y la salud, de donde nació la educación sanitaria, que fue considerada como una especialidad de la pedagogía y que no tuvo avances significativos. A principios de los 80 la educación sanitaria se vio superada por la comunicación para la salud.

Como podemos ver, en ambas génesis prima la funcionalidad (para), y la evolución comparte una finalidad (la salud). No dejan, por tanto, de ser discursos salubristas. Sirva esta propuesta de A. Sendra (2019, p. 37) como una buena definición del campo bajo ese supuesto salubrista (la traducción del inglés es nuestra):

La comunicación en salud es un campo de estudio multidisciplinario y versátil que, utilizando tanto los procesos de comunicación tradicionales como los nuevos, tiene como objetivo mejorar (a través de la promoción, la alfabetización y la educación) las interacciones entre todos los actores que forman parte del sistema de salud (ya sea ciudadanos, pacientes, profesionales de la salud u organizaciones a diferentes niveles); y la calidad, eficiencia y manejo de la salud y la enfermedad (como individuos) y del sistema de salud (como sociedad). 
Aun estando de acuerdo con esta definición, pensamos que comunicación y salud como campo supera lo instrumental y su finalidad salubrista; por ejemplo, contempla también el periodismo sobre salud (una especialización dentro del periodismo científico), la comunicación interna de las instituciones proveedoras de salud, la publicidad (y no solo las campañas) o la señalética, pongamos por caso. De ahí que prefiramos la definición de Silvio Waisbord (2020, p. 32): "Entiendo la comunicación en salud de forma amplia, como un conjunto de esfuerzos por establecer nociones de sentido y significado sobre salud".

De las páginas anteriores se puede desprender que el campo en cuestión es heterogéneo, multidisciplinar, versátil, y que ha de adaptarse a los tiempos y a los contextos en los que se manifiesta. Y eso es también lo que pretende reflejar Comunicación y salud en América Latina: contribuciones al campo.

La obra selecciona 25 comunicaciones de seis congresos de la ALAIC: Bogotá, 2010; Montevideo, 2012; Perú, 2014; Ciudad de México, 2016; Costa Rica, 2018. A esas 25 comunicaciones debemos añadir otros dos textos que van más allá del mero formalismo o protocolo: la "Introducción", realizada por sus editoras, y el "epílogo", que escribe Inesita Soares de Araujo. Por tanto, hemos de hablar, en honor a la verdad, de 27 textos, lo que ya nos da una idea de su ambición.

Como acabamos de escribir, las editoras seleccionaron 25 comunicaciones; ellas mismas dicen que sería erróneo pensar que estas son representativas de lo que se viene escribiendo sobre comunicación y salud en Latinoamérica. No, lo que pretenden es mostrar, por un lado, la heterogeneidad del campo en cuanto a voces, metodologías, enfoques, objetos de estudio y finalidades, y, por otro, que el campo va consolidándose con el transcurso de los años. Y podemos escribir, sin ningún tipo de reparo, que lo consiguen. Este es uno de los grandes logros y valores de la obra: su heterogeneidad.

Que también es lingüística, pues 10 comunicaciones están en su lengua original, el portugués (las 25 incluyen un resumen trilingüe: español, portugués e inglés), y ese respeto por la lengua original de los textos es otro de los aciertos de este libro (por favor, esfuércense un poco, si son capaces de leer el inglés, ¿por qué no van a querer leer el portugués?).

A la par, el que sean textos que ya han pasado por un proceso de selección de pares para ser defendidos en los distintos congresos nos proporciona la garantía de calidad, aunque, como es lógico, cada lector tendrá sus preferidos (también por afinidad con el objeto de estudio o la metodología). Que me perdonen el resto de los autores y autoras, pero por mi parte el primero que leí fue la comunicación de Mariella Silva de Oliveira Costa 
"Comunicar saúde: uma perspectiva teórica". ¿Por qué? Pues porque una de las debilidades de este campo es que falta afianzar la teoría, y esa es una tarea que o vamos haciendo o el campo acabará disuelto por no haberse pensado y conformado; es otra manera de consolidarlo (Terrón, 2010, 2017). Y dado que volvemos a referirnos a la consolidación, debemos agradecer a la ALAIC que tenga en su seno un GT dedicado exclusivamente a la comunicación y salud, algo muy raro entre las asociaciones de comunicación, y que este venga trabajando desde hace tantos años en pro del campo.

También debemos subrayar que en la obra participan 46 autores y autoras de 10 países, entre los que sobresale por número de participantes Brasil, lo cual no es ninguna sorpresa, dado que sin duda alguna es el país de Latinoamérica en el que la comunicación y salud está más consolidada. Por cierto, esa consolidación se consigue con revistas científicas específicas y Brasil cuenta con algunas, y déjenme que mencione a la que considero más relevante: Interface; ${ }^{2}$ en un campo en el cual globalmente prevalecen los estudios cuantitativos, esta revista tiene una especial deferencia por las metodologías cualitativas. Bien es cierto que en español existen dos publicaciones: Revista Española de Comunicación en Salud ${ }^{3}$ y Revista Comunicación y Salud, ${ }^{4}$ pero ambas se editan en Madrid. Animamos a nuestros compañeros y compañeras de Latinoamérica a que pongan todo el empeño en la edición de una revista científica en español que sea una voz periódica de lo que se viene produciendo en el campo en esta amplia región de las Américas.

Por otro lado, cabe decir que en la autoría sobresalen claramente el número de autoras, que, redondeando, suponen dos tercios del total. Esta feminización debería llevarnos a que la visión de género sea, con el tiempo, algo transversal en el campo, sin que ello suponga dejar de focalizar algunos estudios en género y salud. Feminización, por cierto, cada vez más evidente en la comunicación y la salud.

De los 37 autores y autoras de las 25 comunicaciones, 29 provienen de las ciencias de la comunicación, seis de las ciencias sociales (comunicación excluida) y cinco de las ciencias de la salud. Dado que son congresos organizados por una asociación internacional de comunicación, estos números nos parecen previsibles. Ahora bien, ¿hay interdisciplinariedad? Muy poca, otro de los males de la comunicación y salud en cualquier lugar del mundo, por mucho que proclame su interdisciplinariedad como una de sus características. O sea, el libro no deja de ser un reflejo de lo que está ocurriendo en otras regiones del mundo. Ya ven, otra asignatura pendiente del ámbito, y quizás esta sea a la par prioritaria y difícil de alcanzar por la impermeabilidad que suele haber entre los distintos campos académicos.
2 https://interface.org.br/ es/a-revista/

3 https://e-revistas.uc3m.es/ index.php/RECS

4 http:// revistadecomunicacionysalud. es/ 
Durante estos meses estamos viendo una infinidad de artículos que tratan sobre la COVID-19 desde la perspectiva de la comunicación. Si ahondamos en los mismos, nos daremos cuenta de que la gran mayoría no versa sobre salud y que de los que la abordan tangencialmente, casi todos se refieren a la comunicación de riesgos o los peligros de la infodemia y de los bulos y noticias falsas. Es lógico: para escribir con tino sobre comunicación y salud es preciso especializarse o, de otra manera, la salud es el simple recurso para hablar de otros temas. Los que intentamos trabajar en este campo sabemos que investigar en él suele ser más complejo que hacerlo en otros ámbitos de la comunicación. Por fortuna, Comunicación y salud en América Latina: contribuciones al campo es también un expositorio de autoras y de autores que llevan años dedicados a este campo: Mónica Petracci, Janet García, Inesita Soares de Araujo, M. Elena Zermeño (que nos dejó demasiado pronto), Milca Cuberli, etc.

Como es lógico, es imposible realizar una reseña juiciosa de cada una de las 25 comunicaciones en un espacio tan pequeño; así que aconsejamos a los lectores que se acerquen al ejemplar, lean su indice y resúmenes, y comiencen su recorrido por el libro. Libro, les aseguro, al que deberán acudir más de una vez si trabajan el campo de la comunicación y salud o que puede despertarles el deseo de iniciarse en este.

Ya casi terminando, ¿qué esperamos encontrarnos dentro de algunos años en el próximo libro que sin duda compilará el GT5 de Comunicación y Salud de la ALAIC? Que la visión de género sea transversal; interdisciplinariedad; que haya investigaciones sobre la imagen (la gran olvidada en los estudios de comunicación y salud); dedicar más estudios a la salud mental; especialización y colaboración internacional entre los autores; estudios en torno a la calidad de la información sobre salud; dar más importancia a los determinantes sociales; historicidad; descolonizar la elección de teorías y metodologías, por un lado, y, por otro, las miradas y la manera de ver; trabajar por una descentralización de la enunciación; el Otro protagonista y no solo el discurso del Otro que ilustra nuestro punto de vista; una mayor consistencia teórica en las investigaciones a la par que una mayor dedicación de los investigadores al desarrollo teórico de la comunicación y la salud; y hacer un esfuerzo en trabajar sobre la evaluación de campañas. En definitiva, que seamos capaces de avanzar en el desarrollo de la comunicación y salud y que ese desarrollo del campo se transmita con nitidez.

Por último, vale destacar la labor de InCom-UAB Publicacions y de las editoriales similares por hacer posible que obras como esta, vean la luz y sean compartidas libremente lejos de la lógica del mercado editorial. 


\section{Referencias}

Castiel, L. y Álvarez Dardet, C. (2007). La salud persecutoria. Revista Saúde Pública. 41(3), 461-6.

Díaz Rojo, J. A., Morant i Marco, R. y Westall, P. (2006). El culto a la salud y la belleza. La retórica del bienestar. Editorial Biblioteca Nueva.

Hastings Center (2004). Los fines de la medicina. Fundación Víctor Grífols i Lucas.

Huber, M., Knottnerus, A. y Green, L. (2011). How should we define health? BMJ, 343, d4163. http: / / www.bmj.com/content/343/bmj.d4163.full?sid=789abf6e-1669-4b6d-bc4fc8aa94ed1069

Navarro, V. (1998). Concepto actual de salud pública. En L. Castellanos y V. Navarro (Coords.), Salud pública (pp. 49-54). McGraw-Hill. https://ifdcsanluis-slu.infd.edu.ar/sitio/ material-de-estudio-del-ano-2013/upload/navarro.pdf

Petracci, M. y García González, J. (2020). Comunicación y salud en América Latina: contribuciones al campo. InCom-UAB Edicions.

Ramírez Beltrán, L. (1995). Salud pública y comunicación social. Chasqui, 55, 33-37.

Ramírez Leyva, F. (2015). Aproximación al nexo entre comunicación y salud. Implicaciones socio-políticas y periodismo en salud. "Lecciones". Portal Comunicación, Barcelona InCom-UAB.

https: / / incom.uab.cat/ portalcom/aproximacion-al-nexo-entre-comunicacion-y-saludimplicaciones-socio-politicas-y-periodismo-en-salud/?lang=es

Schiavo, R. (2007). Health communication: from theory to practice. Jossey-Bass.

Sendra, A. (2019). Health communication processes in social media: towards a transformative intervention on the information about chronic pain [Tesis de doctorado]. Universidad Rovira i Virgili, Tarragona.

Terrón, J. L. (2017). ¿Qué es salud? Una definición que condiciona nuestro campo de estudio. En U. Cuesta, C. Peñafiel, J. L. Terrón y E. Bustamante (Coords.), Comunicación y salud (pp. 49-60). Dextra.

Terrón, J. L. (2010). Algunas reflexiones sobre la comunicación y salud en España tras cinco años del Observatorio de Comunicación y Salud. Revista Española de Comunicación en Salud, 1(2), 77-97.

Waisbord, Silvio (2020). De la simplicidad a la complejidad: lecciones inconclusas de la pandemia para comunicación y salud pública. Revista ALAIC, 19(35), 30-37. 